\title{
Nominal definition of satellite constellations under the Earth gravitational potential
}

\author{
3 David Arnas · Daniel Casanova
}

5 Received: date / Accepted: date

\begin{abstract}
This work focuses on the definition of satellite constellations whose secular relative distributions are invariant under the perturbation produced by the Earth gravitational potential. This is done by defining the satellite distribution directly in the Earth Centered - Earth Fixed frame of reference and using the along-track time distances between satellites to define the satellite constellation configuration. In addition, in order to expand the possibilities of application of this design methodology, a general transformation between the formulations of Flower Constellations, Walker Constellations, and a relative to Earth formulation based on along-track and cross-track distances between satellites is obtained. This allows not only for a relation between these formulations, but also for the obtainment of the relative-to-Earth distribution of such constellations. Finally, an example of application of these methodologies is presented for a low Earth orbit.
\end{abstract}

Keywords Satellite Constellation · Perturbed dynamics · Nominal design . Mathematical models

\section{Introduction}

A large number of satellite missions require flying over the same regions of the Earth surface periodically for different purposes. One of the most common examples is Earth observation satellites, but there are other uses, such as the

David Arnas

Massachusetts Institute of Technology

Department of Aeronautics and Astronautics

E-mail: arnas@mit.edu

Daniel Casanova

Centro Universitario de la Defensa - Zaragoza

APEDIF - IUMA - Universidad de Zaragoza

E-mail: casanov@unizar.es 
ability to establish communications periodically with certain ground stations, or the study of defined regions of the planet surface that require regional coverage. All these applications are based on satellites that present a particular set of orbital elements related to a feature, the repeating ground-track condition. This property can be easily modeled in a Keplerian formulation with a closed solution. However, if orbital perturbations are considered, the problem becomes more complex and transforms, what once was a simple formulation, into a problem that has no analytical solution.

As a result, several methodologies have appeared over the years to solve this problem with different approaches. For instance, in Wagner's (Wagner, 1991) work, a numerical method based on a semi-major axis correction is used to achieve the repeating ground-track property under the effect produced by the oblateness of the Earth (J2 perturbation). Another example, this time applied to satellite constellations, can be seen in the Flower Constellations (Mortari et al., 2004; Avendaño et al., 2012) where the repeating ground-track property under the effects of $J_{2}$ is taken into account both in the nominal design of the orbits and in their station keeping (Mortari et al., 2014; Casanova et al., 2014c; Arnas et al., 2016a).

In this work, we focus on the nominal definition of repeating groundtrack constellations, that is, constellations whose satellites have the repeating ground-track property and, in addition, are required to share a common ground-track, that is, all satellites will describe the same trajectory from the Earth Centered - Earth Fixed frame of reference. To that end, we propose a constellation design model where the distribution of satellites is performed using the along-track distances in time between the satellites of the constellation. The methodology presented is based on the formulation provided by Arnas et al. (2017a, 2016b), a mathematical model to define satellite constellations that performs the definition of the constellation directly in the ECEF (Earth-Centered, Earth-Fixed) frame of reference using as distribution parameters the along-track and cross-track distances between satellites. Using this relative to Earth formulation allows for a more natural definition of the constellation as related to Earth, and for the inclusion of the effects of orbital perturbations in the initial design of the constellation. In that sense, this formulation presents a different approach to satellite constellation design compared with Flower Constellations (Mortari et al., 2004) and its variants in Lattice (Avendaño et al., 2013; Davis et al., 2013) and Necklace (Arnas, 2018; Casanova et al., 2014a; Arnas et al., 2018, 2017b) formulations, Walker Constellations (Walker, 1984), Draim Elliptic Constellations (Draim, 1987), the Kinematically Regular Satellite Networks (Mozhaev, 1973), the Streets of Coverage (Luders, 1961), or many others (Ulybyshev, 2008; Lo, 1999; Beste, 1978; Ballard, 1980; Wook et al., 2018), where this definition is done in the inertial frame of reference.

To that end, this manuscript introduces a modified formulation of the design model presented in Arnas et al. (2017a) to account for periodic perturbations such as the Earth gravitational potential. This is done by providing a distribution invariant that is used to define the nominal orbits of repeating 
ground-track constellations under the effect of such perturbations. Additionally, and in order to extend this property to other satellite distribution, a general transformation of this formulation with other known satellite constellation designs is provided.

This work is presented as follows. First, we summarize the set of satellite constellation formulations that are used in this work, namely, Walker Constellations, Flower Constellations, 2D Lattice Flower Constellations, 2D Necklace Flower Constellations and a relative to Earth satellite distribution. Second, we introduce a methodology based on the formulation from Arnas et al. (2016b) to define constellations whose satellites share their relative trajectories under the perturbation produced by the Earth gravitational potential. Third, we propose a one to one transformation between the formulations defined by Flower Constellations and Walker Constellations (the most used satellite constellation design to this date), and the ones defined in this work for the cases of repeating ground-track constellations. This is done in order to show the relation between these formulations and to extend the properties of this model to other satellite constellation designs. Fourth, we present an example of an application of this constellation design methodology for a low Earth orbit and study the maintenance of the defined distribution in the long term under the perturbation produced by the Earth gravitational potential.

\section{Preliminaries}

In this section we present a summary of the satellite constellation design formulations that are used in this work. In particular, we deal with the formulations of Walker Constellations, 2D Lattice Flower Constellations, 2D Necklace Flower Constellations and a satellite distribution based on the along-track time distance between the satellites of the constellation.

\subsection{Walker Constellations}

Walker-Delta Constellations (Walker, 1984) are the most well-known satellite constellation design in the literature. They are based on the idea of distributing satellites evenly in a set of equally spaced inertial circular orbits. In this constellation design, all satellites share the nominal values of semi-major axis and inclination. Walker Constellations are defined by the following notation, $i: t / p / f$, being $i$ the inclination of the orbits, $t$ the total number of satellites, $p$ the number of orbital planes of the constellation, and $f \in\{0, \ldots, p-1\}$ a phase parameter that defines the shifting of the distribution in true anomaly from adjacent orbital planes. Particularly, in a Walker Constellation, the right ascension of the ascending node and the mean anomaly follow this distribution:

$$
\begin{aligned}
\Delta \Omega_{i j} & =2 \pi \frac{(i-1)}{p}, \\
\Delta M_{i j} & =2 \pi \frac{p}{t}(j-1)+2 \pi \frac{f}{t}(i-1),
\end{aligned}
$$


where $\Delta \Omega_{i j}$ and $\Delta M_{i j}$ are the right ascension of the ascending node and the mean anomaly of the satellites of the constellation with respect to a reference satellite, and $i$ and $j$ name the satellite in orbit $i$, and position $j$ in that orbit.

\subsection{Flower Constellations}

Flower Constellations (Mortari et al., 2004) is a constellation design methodology that is based on the idea of distributing satellites over a unique space-track in a given reference system. In that sense, they present several similarities with Arnas et al. (2017a) since both deal with the same problem. However, there are two important differences between them. First, Flower Constellations are defined using classical variables (the mean anomaly and the right ascension of the ascending node of the satellites) while Arnas et al. (2017a) uses alongtrack and cross-track time distances between satellites. Second, the resultant distributions generated by Flower Constellations present a set of distribution patterns that are repeated through the space-track, while the other formulation does not impose any restriction in the definition of the along-track distribution.

In the same way as Walker Constellations, a Flower Constellation is characterized for having all satellites with the same value of semi-major axis, eccentricity, inclination and argument of perigee, however, they are not limited to only circular orbits as in the case of Walker Constellations. In a Flower Constellation, the right ascension of the ascending node and the mean anomaly follow this distribution:

$$
\begin{aligned}
\Delta \Omega_{g} & =-2 \pi \frac{F_{n}}{F_{d}}(g-1) \bmod (2 \pi), \\
\Delta M_{g} & =2 \pi \frac{F_{n} N_{p}+F_{d} F_{h}(g)}{F_{d} N_{d}}(g-1) \bmod (2 \pi),
\end{aligned}
$$

where $g \in\{1,2, \ldots\}$ with $g \leq F_{d} N_{d} N_{p}$ names each satellite of the constellation, $F_{d}$ is the number of orbits of the constellation, $F_{n} \in\left\{0,1, \ldots, F_{d}-1\right\}$ with $\operatorname{gcd}\left(F_{n}, F_{d}\right)=1$ is an integer parameter that can be freely chosen, and $F_{h}(g) \in\left\{0,1, \ldots, N_{d}-1\right\}$ is the phasing parameter, which can be changed for each satellite of the constellation.

\subsection{D Lattice Flower Constellations}

2D Lattice Flower Constellations (Avendaño et al., 2013) is a general methodology to generate completely uniform distributions using as a base the Flower Constellation Theory. This means that the constellation configuration is the same no matter the satellite selected as the reference. In general, 2D Lattice Flower Constellations distribute satellites in different space-tracks (contrary to what happened in the original Flower Constellations where all satellites where located in a common space-track) containing an equal number of satellites. In 
a 2D Lattice Flower Constellation, satellites share the same semi-major axis, eccentricity, inclination and argument of perigee, while their right ascension of the ascending node and mean anomaly follow this distribution:

$$
\begin{aligned}
\Delta \Omega_{i j} & =\frac{2 \pi}{L_{\Omega}}(i-1) \quad \bmod (2 \pi), \\
\Delta M_{i j} & =\frac{2 \pi}{L_{M}}(j-1)-\frac{2 \pi}{L_{M}} \frac{L_{M}}{L_{\Omega}}(i-1) \bmod (2 \pi),
\end{aligned}
$$

where $L_{\Omega}$ is the number of orbits of the constellation, $L_{M}$ is the number of satellites per orbit, and $i \in\left\{1, \ldots, L_{\Omega}\right\}$ and $j \in\left\{1, \ldots, L_{M}\right\}$ name each satellite of the constellation. Finally, $L_{M \Omega} \in\left\{0,1, \ldots, L_{\Omega}-1\right\}$ is the combination number, an integer parameter that allows to shift the distribution between different orbital planes. As it can be seen from Eqs. (1) and (3), Walker Constellations constitute a particularization for circular orbits of the more general 2D Lattice Flower Constellations.

\subsection{D Necklace Flower Constellations}

2D Necklace Flower Constellations (Arnas et al., 2018) are based on the idea of generating a fictitious constellation based on the 2D Lattice Flower Constellations formulation, which is a completely uniform distribution, and then select, from the set of available positions already defined, the subset of satellites that fulfills a series of mission requirements. When dealing with uniform distributions, 2D Necklace Flower Constellations are related to 2D Lattice Flower Constellations through:

$$
\begin{aligned}
(i-1) & =\mathcal{G}_{\Omega}-1 \bmod \left(L_{\Omega}\right), \\
(j-1) & =\mathcal{G}_{M}-1+S_{M \Omega}\left(\mathcal{G}_{\Omega}-1\right) \bmod \left(L_{M}\right),
\end{aligned}
$$

where $\mathcal{G}_{\Omega}$ and $\mathcal{G}_{M}$ represent the necklaces in the right ascension of the ascending node and the mean anomaly respectively, and $S_{M \Omega}$ is the shifting parameter that relates the movement of the necklace in the mean anomaly with the orbital plane considered. Under this definition, $\mathcal{G}_{\Omega}$ is a subset from $\mathcal{G}_{\Omega} \in\left\{1,2, \ldots, L_{\Omega}\right\}$ which represents a subset of orbital planes selected from the complete lattice configuration. In a similar manner, $\mathcal{G}_{M}$ is a subset of elements from $\mathcal{G}_{M} \in\left\{1,2, \ldots, L_{M}\right\}$ and represents a subset of positions from the set of available positions in each orbit. This means that the formulation is able to define directly which are the actual occupied positions in the constellation without requiring to define all the positions from the complete lattice. In addition, and if a complete uniform distribution is required, the shifting parameter has to fulfill the following relation (Arnas et al., 2018):

$$
\operatorname{Sym}\left(\mathcal{G}_{M}\right) \mid S_{M \Omega} L_{\Omega}-L_{M \Omega},
$$

which reads $\operatorname{Sym}\left(\mathcal{G}_{M}\right)$ divides $\left(S_{M \Omega} L_{\Omega}-L_{M \Omega}\right)$; where $\operatorname{Sym}\left(\mathcal{G}_{M}\right)$ is the symmetry of the necklace in the mean anomaly, that is, the minimum number of 
rotations that the necklace has to perform in the available positions to generate the same distribution. For instance, the necklace $\mathcal{G}_{M}=\{1,3,5\} \in \mathbb{N}_{6}$ has $\operatorname{Sym}\left(\mathcal{G}_{M}\right)=2$ since $\mathcal{G}_{M}=\{1,3,5\} \equiv\{3,5,7\} \bmod (6)$.

\subsection{Relative to Earth satellite distribution}

We define repeating ground-track constellations as the constellations whose satellites share a set of defined repeating ground-tracks. In order to achieve this condition, the dynamic of satellites must fulfill a compatibility relation with the rotation of the Earth given by:

$$
T_{c}=N_{p} T_{\Omega}=N_{d} T_{\Omega G}
$$

where $T_{c}$ is the period of the repeating cycle, $T_{\Omega}$ is the nodal period of the orbit, $T_{\Omega G}$ is the nodal period of Greenwich, $N_{p}$ is the number of orbital revolutions of the satellite to cycle repetition, and $N_{d}$ is the number of days to cycle repetition. Note that $N_{p}$ and $N_{d}$ are coprime numbers to avoid duplicate definitions of the same configurations using Eq. (6) (Avendaño et al., 2012). In general, this condition is applied individually for each satellite of the constellation obtaining a repeating ground-track constellation. However, in this work we approach this problem from a different prespective using the formulation seen in Arnas et al. (2016b). This new approach is based on including the periodic orbital perturbations directly on the nominal design of the constellation.

Arnas et al. (2017a) proposes a satellite constellation design based on the idea of defining a series of space-tracks (or relative trajectories) where all the satellites of the constellation are located. The particularity of this formulation is that the distribution is defined based on the along-track time distances and cross-track separation between satellites. That way, and for a non-perturbed dynamical model, the distribution of the constellation can be defined by:

$$
\begin{aligned}
\Delta \Omega_{k q} & =\Delta \Omega_{k}-\omega_{\oplus}\left(t_{k q}-t_{0}\right), \\
\Delta M_{k q} & =n\left(t_{k q}-t_{0}\right),
\end{aligned}
$$

where the parameters $(k, q)$ relate to a given spacecraft in the space-track $k$ and position $q$ in that space-track; $\Delta \Omega_{k q}$ and $\Delta M_{k q}$ are the right ascension of the ascending node and the mean anomaly of the satellites of the constellation with respect to a given reference; $\Delta \Omega_{k}$ is the cross-track angular distance of the space-tracks with respect to the reference, $\omega_{\oplus}$ is the spin rate of the Earth, $n$ is the mean motion of the satellites, and $\left(t_{k q}-t_{0}\right)$ is the along-track time distance of each satellite with respect to a reference. On the other hand, the values of the semi-major axis $a$, eccentricity $e$, inclination $i$ and argument of perigee $\omega$ are shared by all the satellites of the constellation.

Additionally, and when dealing with repeating ground-track orbits, it is possible to relate the dynamics of satellites with the movement of the Earth 
using Eq. (6):

$$
T_{c}=N_{p} \frac{2 \pi}{n}=N_{d} \frac{2 \pi}{\omega_{\oplus}},
$$

which can be introduced in Eq. (7) to obtain the following expression:

$$
\begin{aligned}
\Delta \Omega_{k q} & =\Delta \Omega_{k}-2 \pi N_{d} \frac{\left(t_{k q}-t_{0}\right)}{T_{c}}, \\
\Delta M_{k q} & =2 \pi N_{p} \frac{\left(t_{k q}-t_{0}\right)}{T_{c}},
\end{aligned}
$$

where $\left(t_{k q}-t_{0}\right) \in\left[0, T_{c}\right)$. Note that now $T_{c}$ is the parameter that defines the general dynamic of the constellation. This expression can define any constellation distribution where all satellites have the same repetition cycle $T_{c}$. Moreover, it is interesting to study also the case where all satellites of the constellation share the same ground-track, that is, $k=1$. For those cases, Eq. (9) can be simplified into:

$$
\begin{aligned}
\Delta \Omega_{q} & =-2 \pi N_{d} \frac{\left(t_{q}-t_{0}\right)}{T_{c}}, \\
\Delta M_{q} & =2 \pi N_{p} \frac{\left(t_{q}-t_{0}\right)}{T_{c}},
\end{aligned}
$$

where we have changed the sub-indexes to $q$ in order to make it clear that only one ground-track is considered for the distribution. Furthermore, if a uniform distribution of satellites is required along the ground-track, we can define the constellation by means of a distribution parameter $q \in\left\{1, \ldots, N_{s t}\right\}$ where $N_{s t}$ is the number of satellites of the constellation. That way, and since the distribution is uniform, the along-track configuration can be defined by:

$$
t_{q}-t_{0}=\frac{(q-1)}{N_{s t}} T_{c},
$$

which introduced in Eq. (10) leads to:

$$
\begin{aligned}
\Delta \Omega_{q} & =-2 \pi N_{d} \frac{(q-1)}{N_{s t}}, \\
\Delta M_{q} & =2 \pi N_{p} \frac{(q-1)}{N_{s t}},
\end{aligned}
$$

where $q$ names each satellite of the constellation. Note that although Eq. (12) is a general formulation that allows to generate satellite distributions based on a common ground-track, this kind of distribution can be obtained with many other formulations. 


\section{Designing repeating ground-track constellations}

In Section 2 we summarized the formulations of some well known satellite constellation design models under a non-perturbed model. The idea of this section is to develop a mathematical model which includes the Earth gravitational potential in its formulation, identifying an invariant in the distribution under such perturbation. In order to do that, we first study the evolution of the system under the Earth gravitational potential, and from it, we propose a modified satellite constellation definition based on the formulation presented in Eq. (12) and evaluate its long term dynamic.

\subsection{Perturbed dynamic}

When orbital perturbations are considered, it is useful to take their effects into account when performing the nominal distribution of the constellation. In particular, Eq. (9) can be written in terms of the nodal periods. Using the relations presented in Eqs. (6) and (8) the following expression can be obtained:

$$
\begin{aligned}
\Delta \Omega_{k q} & =\Delta \Omega_{k}-\frac{2 \pi}{T_{\Omega G}}\left(t_{k q}-t_{0}\right), \\
\Delta M_{k q} & =\frac{2 \pi}{T_{\Omega}}\left(t_{k q}-t_{0}\right),
\end{aligned}
$$

which relates the distribution to the nodal periods associated with the constellation. However, due to orbital perturbations, the reference position where the mean anomaly is defined, the perigee of the orbit, can change, and thus, this effect must be taken into account. In order to overcome this difficulty, the constellation is defined related to the Earth Equator, instead of the apogee of the orbits, that is:

$$
\begin{aligned}
& \Delta \Omega_{k q}=\Delta \Omega_{k}-\frac{2 \pi}{T_{\Omega G}}\left(t_{k q}-t_{0}\right), \\
& \Delta \chi_{k q}=\Delta M_{k q}+\Delta \omega_{k q}=\frac{2 \pi}{T_{\Omega}}\left(t_{k q}-t_{0}\right)+\Delta \omega_{k q},
\end{aligned}
$$

where we define $\Delta \chi_{k q}=\Delta M_{k q}+\Delta \omega_{k q}$ as the mean argument of latitude of each satellite with respect to a given reference. It is important to note that, for a repeating ground-track constellation, if no orbital perturbations are considered, every satellite must have the same argument of perigee, and thus, $\Delta \omega_{k q}=0$. Equation (14) represents a generalization of Eq. (7) for repeating ground-track constellations under orbital perturbations since it only depends on the resultant dynamic with respect to the movement of the Earth.

Moreover, the nodal period of the orbit $\left(T_{\Omega}\right)$ and the nodal period of Greenwich $\left(T_{\Omega G}\right)$ are also affected by orbital perturbations, transforming the relation showed in Eq. (8) into:

$$
T_{c}=N_{p} \frac{2 \pi}{n_{k q}+\dot{M}_{k q}^{o}+\dot{\omega}_{k q}}=N_{d} \frac{2 \pi}{\omega_{\oplus}-\dot{\Omega}_{k q}},
$$


where $n_{k q}$ is the mean motion, $\dot{M}_{k q}^{o}$ is the secular variation of the mean argument with respect to the mean motion, $\dot{\omega}_{k q}$ is the secular variation of the argument of perigee, and $\dot{\Omega}_{k q}$ is the secular variation of the right ascension of the ascending node of each of the satellites of the constellation. By introducing the perturbed values of the nodal periods into Eq. (14), we obtain:

$$
\begin{aligned}
\Delta \Omega_{k q} & =\Delta \Omega_{k}-\omega_{\oplus}\left(t_{k q}-t_{0}\right)+\dot{\Omega}_{k q}\left(t_{k q}-t_{0}\right), \\
\Delta \chi_{k q} & =n_{k q}\left(t_{k q}-t_{0}\right)+\left(\dot{M}_{k q}^{o}+\dot{\omega}_{k q}\right)\left(t_{k q}-t_{0}\right),
\end{aligned}
$$

which clearly shows that the distribution must take into account the rotation of the orbits in their orbital planes and also the drift that the orbital planes experience from the reference time in order to maintain the sharing of the ground-tracks of the constellation. Moreover, if the relations from Eq. (15) are used in Eq. (16), we can derive the following distribution under orbital perturbations:

$$
\begin{aligned}
\Delta \Omega_{k q} & =\Delta \Omega_{k}-2 \pi N_{d} \frac{\left(t_{k q}-t_{0}\right)}{T_{c}}, \\
\Delta \chi_{k q} & =2 \pi N_{p} \frac{\left(t_{k q}-t_{0}\right)}{T_{c}},
\end{aligned}
$$

which is equivalent as the one obtained in Eq. (9). This implies that the alongtrack distribution can be maintained from the non-perturbed definition to the nominal distribution under orbital perturbations. The same can be said for Eq. (10), as it is a particular case of application. Note that the inertial distribution must change when dealing with a perturbed model since $T_{c}$ depends on the orbital perturbations considered.

\subsection{Constellation definition}

Equation (16) would lead, in general, to a difficult process in order to obtain compatible constellations that fulfill the distribution under orbital perturbations. This is due to the fact that the secular variation of the orbital elements depends on the initial position of each satellite. However, there is an alternative approach to solve this problem when dealing with the perturbations produced by the Earth gravitational potential, which is the case when defining the nominal orbits of a constellation. In particular, we know that from the ECEF frame of reference, the gravitational field of the Earth can be approximated as independent with time. This means that the dynamic of satellites only depends on the trajectories that they follow in this reference system, and not on the moment when they fly over these trajectories. In other words, $\dot{\Omega}_{k q}=\dot{\Omega}_{k}, n_{k q}=n_{k}, \dot{M}_{k q}^{o}=\dot{M}_{k}^{o}$ and $\dot{\omega}_{k q}=\dot{\omega}_{k}$. Therefore, Eq. (16) can be rewritten in terms of the different space tracks in the ECEF frame of reference:

$$
\begin{aligned}
\Delta \Omega_{k q} & =\Delta \Omega_{k}-\omega_{\oplus}\left(t_{k q}-t_{0}\right)+\dot{\Omega}_{k}\left(t_{k q}-t_{0}\right), \\
\Delta \chi_{k q} & =n_{k}\left(t_{k q}-t_{0}\right)+\left(\dot{M}_{k}^{o}+\dot{\omega}_{k}\right)\left(t_{k q}-t_{0}\right),
\end{aligned}
$$


where the sub-indexes in $k$ relate to each space-track of the constellation. Thus, a set of satellites that share a particular space-track from the ECEF frame of reference (even if it is not closed), and under the Earth gravitational potential, will continue to share their space-track over the course of their orbits. This property is used in here in combination with the formulation presented in Section 2.5 to perform the nominal definition of the constellation.

That way, if we focus on a particular space-track of the constellation, we can define a leading satellite (which is not required to be a real satellite of the constellation) and use it to define a space-track related to the ECEF frame of reference for a given time interval. This is done by performing a propagation of this satellite under the Earth gravitational potential. Then, taking any point defined during this propagation in the ECEF frame of reference and assigning it to a satellite of the constellation leads to a distribution whose satellites share the same space-track over time. In other words, the distribution of satellites in the constellation follow these relations (Arnas et al., 2016b):

$$
\begin{aligned}
\mathbf{x}_{\mathbf{q}}\left(t_{0}\right) & =\mathbf{x}_{\mathbf{l s}}\left(t_{q}\right), \\
\mathbf{v}_{\mathbf{q}}\left(t_{0}\right) & =\mathbf{v}_{\mathbf{l s}}\left(t_{q}\right),
\end{aligned}
$$

where $\mathbf{x}_{\mathbf{q}}\left(t_{0}\right)$ and $\mathbf{v}_{\mathbf{q}}\left(t_{0}\right)$ are the position and velocity of satellite $q$ in the ECEF frame of reference at the initial time $\left(t_{0}\right)$, while $\mathbf{x}_{\mathbf{l s}}\left(t_{q}\right)$ and $\mathbf{v}_{\mathbf{l s}}\left(t_{q}\right)$ are the position and velocity in the ECEF of the leading satellite for that space-track at time $t_{q}$. This process is then continued by defining a leading satellite for each space-track of the constellation and generating the satellite distribution related to it following the same methodology.

Thus, the mean evolution of the right ascension of the ascending node and the mean argument of latitude for the leading satellite in time $t_{k q}$, when considering repeating ground-track orbits, is provided by:

$$
\begin{aligned}
& \Omega_{l s}\left(t_{k q}\right)=\Omega_{l s}\left(t_{0}\right)+\dot{\Omega}_{l s}\left(t_{k q}-t_{0}\right), \\
& \chi_{l s}\left(t_{k q}\right)=\chi_{l s}\left(t_{0}\right)+n_{l s}\left(t_{k q}-t_{0}\right)+\left(\dot{M}_{l s}^{o}+\dot{\omega}_{l s}\right)\left(t_{k q}-t_{0}\right),
\end{aligned}
$$

where the sub-index $l s$ relate to the leading satellite of each space-track. Equation (20) represents the same distribution as the one defined in Eq. (18) except for a rotation in the right ascension of the ascending node corresponding to the difference in the spin rates of the ECEF and inertial frames of reference. Therefore, each leading satellite is able to define the positions of all satellites that share its space-track under the perturbation produced by the Earth gravitational potential.

\subsection{Evolution of the distribution}

Now, we will study the evolution of this kind of distribution under the Earth gravitational potential. To that end, we compare the dynamic of a leading satellite with one of the satellites of the constellation that is located in the same relative to Earth trajectory at an along-track distance of $t_{q}$. Let $t_{f}$ be 
a given general instant in which the satellite distribution is studied. At that time, the leading satellite will have the following secular orbital elements:

$$
\begin{aligned}
\Omega_{l s}\left(t_{f}\right) & =\Omega_{l s}\left(t_{0}\right)+\dot{\Omega}_{l s}\left(t_{f}-t_{0}\right) \\
\chi_{l s}\left(t_{f}\right) & =\chi_{l s}\left(t_{0}\right)+n_{l s}\left(t_{f}-t_{0}\right)+\left(\dot{M}_{l s}^{o}+\dot{\omega}_{l s}\right)\left(t_{f}-t_{0}\right),
\end{aligned}
$$

On the other hand, the evolution of the secular values of the orbital elements for the second satellite $(q)$ can be obtained through:

$$
\begin{aligned}
\Omega_{q}\left(t_{f}\right) & =\Omega_{q}\left(t_{0}\right)+\dot{\Omega}_{l s}\left(t_{f}-t_{0}\right), \\
\chi_{q}\left(t_{f}\right) & =\chi_{q}\left(t_{0}\right)+n_{l s}\left(t_{f}-t_{0}\right)+\left(\dot{M}_{l s}^{o}+\dot{\omega}_{l s}\right)\left(t_{f}-t_{0}\right),
\end{aligned}
$$

which compared to the evolution of the leading satellite leads to:

$$
\begin{gathered}
\Delta \Omega_{q}\left(t_{f}\right)=\Omega_{q}\left(t_{f}\right)-\Omega_{l s}\left(t_{f}\right)=\Omega_{q}\left(t_{0}\right)-\Omega_{l s}\left(t_{0}\right)=\Delta \Omega_{q}\left(t_{0}\right), \\
\Delta \chi_{q}\left(t_{f}\right)=\chi_{q}\left(t_{f}\right)-\chi_{l s}\left(t_{f}\right)=\chi_{q}\left(t_{0}\right)-\chi_{l s}\left(t_{0}\right)=\Delta \chi_{q}\left(t_{0}\right) .
\end{gathered}
$$

This means that the distribution of the constellation is maintained regarding its secular values.

Therefore, by following the satellite distribution provided by:

$$
\begin{aligned}
\Delta \Omega_{k q} & =\Delta \Omega_{k}-\frac{2 \pi}{T_{\Omega G}}\left(t_{k q}-t_{0}\right), \\
\Delta \chi_{k q} & =\Delta \omega_{k q}+\frac{2 \pi}{T_{\Omega}}\left(t_{k q}-t_{0}\right),
\end{aligned}
$$

it is possible to perform the nominal definition of a repeating ground-track constellation under the perturbation produced by the Earth gravitational potential. Moreover, this methodology shows that using a constellation definition from the ECEF frame of reference provides important advantages when dealing with the nominal design of the orbits under such perturbations. In particular, it allows to include the effects of the gravitational potential of the Earth directly in the nominal definition of the constellation; and it provides a very simple methodology to distribute satellites under this dynamic. Note also that the process introduced in this section can be applied to the definition of constellations around any celestial body that presents a gravitational field that can be considered as time invariant in a given reference frame.

\subsection{Constellation definition by a series expansion}

In previous subsections, we have dealt with a study of the evolution of satellite distributions over time by taking into account the secular variations of the orbital variables. However, it is also possible to reach the same conclusions by taking into account the complete series expansion of the orbital variables considered. That way, we can rewrite Eq. (18) by including the complete series 
expansion of the orbital variables of the satellite distribution under the Earth gravitational potential:

$$
\begin{aligned}
\Delta \Omega_{k q} & =\Delta \Omega_{k}-\omega_{\oplus}\left(t_{k q}-t_{0}\right)+\sum_{i=1}^{\infty} \frac{1}{i !} \frac{d^{i} \Omega_{k}}{d t^{i}}\left(t_{k q}-t_{0}\right)^{i} \\
\Delta \chi_{k q} & =n_{k}\left(t_{k q}-t_{0}\right)+\left(\dot{M}_{k}^{o}+\dot{\omega}_{k}\right)\left(t_{k q}-t_{0}\right)+ \\
& +\sum_{i=2}^{\infty} \frac{1}{i !}\left[\frac{d^{i-1} n_{k}}{d t^{i-1}}+\frac{d^{i}\left(M_{k}^{o}+\omega_{k}\right)}{d t^{i}}\right]\left(t_{k q}-t_{0}\right)^{i},
\end{aligned}
$$

and then, relate them with the dynamic of a leading satellite of the constellation as done in Eq. (20):

$$
\begin{aligned}
\Omega_{l s}\left(t_{k q}\right) & =\Omega_{l s}\left(t_{0}\right)+\sum_{i=1}^{\infty} \frac{1}{i !} \frac{d^{i} \Omega_{l s}}{d t^{i}}\left(t_{k q}-t_{0}\right)^{i} \\
\chi_{l s}\left(t_{k q}\right) & =\chi_{l s}\left(t_{0}\right)+n_{l s}\left(t_{k q}-t_{0}\right)+\left(\dot{M}_{l s}^{o}+\dot{\omega}_{l s}\right)\left(t_{k q}-t_{0}\right)+ \\
& +\sum_{i=2}^{\infty} \frac{1}{i !}\left[\frac{d^{i-1} n_{k}}{d t^{i-1}}+\frac{d^{i}\left(M_{l s}^{o}+\omega_{l s}\right)}{d t^{i}}\right]\left(t_{k q}-t_{0}\right)^{i},
\end{aligned}
$$

which leads to the following expressions:

$$
\begin{aligned}
\Delta \Omega_{k q} & +\omega_{\oplus}\left(t_{k q}-t_{0}\right)-\Delta \Omega_{k}=\Omega_{l s}\left(t_{k q}\right)-\Omega_{l s}\left(t_{0}\right)=\sum_{i=1}^{\infty} \frac{1}{i !} \frac{d^{i} \Omega_{l s}}{d t^{i}}\left(t_{k q}-t_{0}\right)^{i} \\
\Delta \chi_{k q} & =\chi_{l s}\left(t_{k q}\right)-\chi_{l s}\left(t_{0}\right)=n_{l s}\left(t_{k q}-t_{0}\right)+\left(\dot{M}_{l s}^{o}+\dot{\omega}_{l s}\right)\left(t_{k q}-t_{0}\right)+ \\
& +\sum_{i=2}^{\infty} \frac{1}{i !}\left[\frac{d^{i-1} n_{k}}{d t^{i-1}}+\frac{d^{i}\left(M_{l s}^{o}+\omega_{l s}\right)}{d t^{i}}\right]\left(t_{k q}-t_{0}\right)^{i} .
\end{aligned}
$$

This represents an equivalent constellation distribution based solely on the trajectory defined by the leading satellite in its dynamic under the Earth gravitational potential. In particular, we can reorder the expression to obtain:

$$
\begin{aligned}
\Delta \Omega_{k q} & =\Delta \Omega_{k}-\omega_{\oplus}\left(t_{k q}-t_{0}\right)+\sum_{i=1}^{\infty} \frac{1}{i !} \frac{d^{i} \Omega_{l s}}{d t^{i}}\left(t_{k q}-t_{0}\right)^{i} \\
\Delta \chi_{k q} & =n_{l s}\left(t_{k q}-t_{0}\right)+\left(\dot{M}_{l s}^{o}+\dot{\omega}_{l s}\right)\left(t_{k q}-t_{0}\right)+ \\
& +\sum_{i=2}^{\infty} \frac{1}{i !}\left[\frac{d^{i-1} n_{l s}}{d t^{i-1}}+\frac{d^{i}\left(M_{l s}^{o}+\omega_{l s}\right)}{d t^{i}}\right]\left(t_{k q}-t_{0}\right)^{i},
\end{aligned}
$$

which is equivalent to Eq. (25) since the perturbation considered only depends on the position of satellites in the ECEF frame of reference. This allows also to obtain the instantaneous values of the orbital distribution at any instant by means of the perturbed orbit of the leading satellite. 


\section{From Flower Constellations to relative to Earth distributions}

In this section we deal with the problem of transforming distributions which are based on the Flower Constellation Theory (ECI - defined) into the formulation provided by Eq. (12) (ECEF - defined). This has two objectives. First, to provide a one to one correspondence between existing satellite constellation design formulations and the formulation used in this work for the case of repeating ground-track constellations. This allows, for instance, to obtain the revisiting times of the satellites of a constellation since the relative positions of the satellites in the ECEF frame of reference are known. Second, this transformation allows extending the properties under the Earth gravitational potential that the formulation presented in this work provides to other constellation definitions. In that sense, we select Flower Constellations as a reference design since they represent the generalization of the most common satellite constellation designs (Davis et al., 2012), particularly, they are a generalization of Walker Constellations (Walker, 1984), Dufour Constellations (Dufour, 2003) and Draim Constellations (Draim, 1987).

The satellite constellation designs that are considered in this manuscript are the following: the Flower Constellations, the 2D Lattice Flower Constellations, the 2D Necklace Flower Constellations and the Walker-Delta Constellations. In that respect, this section focuses on constellations distributed in only one ground-track. This is done since the original Flower Constellation are limited to this kind of design, and also due to the fact that having all the satellites in a common ground-track is a very extended practice that is worthwhile to study independently. To that end, the transformation and parameter conditions that these satellite constellation designs must meet are included. Note that Walker-Delta Constellations are a particularization of 2D Lattice Flower Constellations for circular orbits. However, we have also included this satellite constellation methodology in this work due to its importance in the literature.

\subsection{Flower Constellations}

We relate the distribution defined by Eq. (2) with a uniform distribution in the ECEF frame of reference, represented by Eq. (12) (note that Flower Constellations are distributed in only one ground-track). To that end, and since we want to consider all possible combinations of Flower Constellations, we define a number of possible positions distributed uniformly in a ground-track equal to $N_{s t}=F_{d} N_{d} N_{p}$. That way, and equating Eqs. (2) and (12) we obtain:

$$
\begin{aligned}
& \Delta \Omega_{g}=\Delta \Omega_{q} \quad \bmod (2 \pi), \\
& \Delta M_{g}=\Delta M_{q} \quad \bmod (2 \pi),
\end{aligned}
$$

which after some elemental operations (multiplying by $N_{p} F_{d} / 2 \pi$ ) leads to:

$$
\begin{aligned}
& (q-1)=F_{n} N_{p}(g-1) \quad \bmod \left(N_{p} F_{d}\right), \\
& (q-1)=\left(F_{n} N_{p}+F_{d} F_{h}(g)\right)(g-1) \quad \bmod \left(N_{p} F_{d}\right),
\end{aligned}
$$


which due to its modular character can be expressed as:

$$
\begin{aligned}
& (q-1)=F_{n} N_{p}(g-1)+A F_{d} N_{p}, \\
& (q-1)=\left(F_{n} N_{p}+F_{d} F_{h}(g)\right)(g-1)+B F_{d} N_{d},
\end{aligned}
$$

where $A$ and $B$ are two unknown integers. By subtracting the two equations in Eq. (31) and performing some operations, we obtain:

$$
F_{h}(g)(g-1)=A N_{p}-B N_{d},
$$

which always has a solution for each possible combination of parameters, since $N_{p}$ and $N_{d}$ are always relative prime between them (Mordell, 1969). That way, once $A$ and $B$ are determined and substituted them into Eq. (31), the relative positions $(q)$ of all the satellites of the constellation are obtained. Then, using that result, the along-track distribution of the constellation is provided by Eq. (11), which could be used, for instance, to compute the revisiting time of the subsatellite points (points of intersection between the radio vector of each satellite and the Earth surface) of the constellation by the sole use of integer operations.

\subsection{D Lattice Flower Constellations}

In general, 2D Lattice Flower Constellations generate distributions based on one or several different ground-tracks. As a first case of study, we focus on designing 2D Lattice Flower Constellations in such a way that all satellites share the same ground-track. This requires to impose some conditions in the distribution parameters: number of satellites per orbit $\left(L_{M}\right)$, number of orbits $\left(L_{\Omega}\right)$ and combination number $L_{M \Omega}$. In particular, by equating the right ascension of ascending node from Eqs. (12) and (3) we obtain:

$$
-2 \pi N_{d} \frac{(q-1)}{N_{s t}}=2 \pi \frac{(i-1)}{L_{\Omega}}+2 \pi C,
$$

where $C$ is an unknown integer resultant from the modular arithmetic intrinsic in the right ascension of the ascending node, and $N_{s t}=L_{\Omega} L_{M}$ since both constellations must present the same number of satellites. Then, after some simple operations, Eq. (33) leads to:

$$
L_{M}(i-1)+\left(L_{\Omega} L_{M}\right) C=-N_{d}(q-1),
$$

which is a Diophantine equation (Mordell, 1969) where a solution exists if and only if $\operatorname{gcd}\left(L_{M}, L_{\Omega} L_{M}\right) \mid N_{d}$, which reads $\operatorname{gcd}\left(L_{M}, L_{\Omega} L_{M}\right)$ divides $N_{d}$. This condition can be expressed in a simpler manner as $L_{M} \mid N_{d}$, that is, the number of satellites per orbit $L_{M}$ must be a divisor of $N_{d}$. Condition $L_{M} \mid N_{d}$ imposes a constraint in the selection of the satellites per orbit of the constellation that is the result of the different possibilities that uniform configurations can present in their distribution over the nodes of an inertial orbit. 
$L_{M \Omega}(\lambda)=L_{M \Omega}(0)+\lambda \frac{L_{\Omega} L_{M}}{\operatorname{gcd}\left(N_{d}, L_{\Omega} L_{M}\right)}=L_{M \Omega}(0)+\lambda \frac{L_{\Omega} L_{M}}{L_{M}}=L_{M \Omega}(0)+\lambda L_{\Omega}$,

On the other hand, in order for a given constellation to have all its satellites in the same ground-track, the constellation distribution must fulfill the following condition (Avendaño et al., 2013):

$N_{p} \Delta \Omega_{i j}+N_{d} \Delta M_{i j}=0 \bmod (2 \pi) \quad \Longrightarrow \quad N_{p} \Delta \Omega_{i j}+N_{d} \Delta M_{i j}+2 \pi D=0$,

being $D$ an unknown integer. Then, by substituting Eq. (3) into the previous expression, we obtain:

$$
2 \pi N_{p} \frac{(i-1)}{L_{\Omega}}+2 \pi N_{d}\left(\frac{j-1}{L_{M}}-\frac{L_{M \Omega}(i-1)}{L_{\Omega} L_{M}}\right)+2 \pi D=0,
$$

which after some elemental operations leads to:

$$
N_{d} L_{\Omega}(j-1)+\left(L_{\Omega} L_{M}\right) D=-\left(N_{p} L_{M}-N_{d} L_{M \Omega}\right)(i-1)
$$

where, in order for the solution to exist, $\operatorname{gcd}\left(N_{d} L_{\Omega}, L_{\Omega} L_{M}\right) \mid\left(N_{p} L_{M}-N_{d} L_{M \Omega}\right)$. Taking into account that $\operatorname{gcd}\left(N_{d} L_{\Omega}, L_{\Omega} L_{M}\right)=L_{\Omega} \operatorname{gcd}\left(N_{d}, L_{M}\right)$ and considering that $L_{M} \mid N_{d}$ as previously stated, we conclude that $\operatorname{gcd}\left(N_{d} L_{\Omega}, L_{\Omega} L_{M}\right)=$ $L_{\Omega} L_{M}$. Consequently, and in order for a solution to exist, $L_{\Omega} L_{M}$ must divide $\left(N_{p} L_{M}-N_{d} L_{M \Omega}\right)$. Thus, the value of the combination number $L_{M \Omega}$ is a solution of the following Diophantine equation:

$$
N_{d} L_{M \Omega}+\left(L_{\Omega} L_{M}\right) E=N_{p} L_{M},
$$

being $E$ an unknown integer. The solution of this Diophantine equation exists if and only if:

$$
\operatorname{gcd}\left(N_{d}, L_{\Omega} L_{M}\right)\left|N_{p} L_{M} \quad \Longleftrightarrow \quad \operatorname{gcd}\left(\frac{N_{d}}{L_{M}}, L_{\Omega}\right)\right| N_{p}
$$

Since $\operatorname{gcd}\left(N_{d}, N_{p}\right)=1$ and $L_{M} \mid N_{d}$, it can be concluded that $\operatorname{gcd}\left(\frac{N_{d}}{L_{M}}, L_{\Omega}\right)=$ 1, which means that the number of orbits of the constellation $\left(L_{\Omega}\right)$ has to be coprime with $N_{d} / L_{M}$, which also implies that $\operatorname{gcd}\left(N_{d}, L_{\Omega} L_{M}\right)=L_{M}$. Therefore, the possible values of the combination number $L_{M} \Omega$ provided by Eq. (38) are: where $\lambda$ is any integer number and $L_{M \Omega}(0)$ is a particular solution of Eq. (38). As it can be seen, the value of $L_{M \Omega}$ is unique, since the combination numbers are defined such that $L_{M \Omega} \in\left\{0,1, \ldots, L_{\Omega}-1\right\}$ to avoid duplicities in the formulation (Arnas et al., 2018). Thus, all conditions that 2D Lattice Flower Constellations must fulfill in order to generate a repeating ground-track constellation are known:

$$
L_{M} \mid N_{d}, \quad \operatorname{gcd}\left(\frac{N_{d}}{L_{M}}, L_{\Omega}\right)=1, \quad \text { and } \quad\left(N_{d} L_{M \Omega}-N_{p} L_{M}\right) \mid L_{\Omega} L_{M} .
$$


Now, we plan to relate the resultant distribution with the configuration 9 generated by Eq. (12). In that sense, since the distributions from both formu450 lations are completely uniform, the number of available positions in the ECEF 1 must be $N_{s t}=L_{\Omega} L_{M}$, that is, the number of satellites of the $2 \mathrm{D}$ Lattice Flower Constellation. Then, by equating Eqs. (3) and (12) we obtain:

$$
\frac{2 \pi}{L_{M}}(j-1)-\frac{2 \pi}{L_{M}} \frac{L_{M \Omega}}{L_{\Omega}}(i-1)=2 \pi N_{p} \frac{(q-1)}{N_{s t}} \bmod (2 \pi),
$$

which after some elemental operations, and knowing that the number of satellites is $N_{s t}=L_{\Omega} L_{M}$, leads to:

$$
N_{p}(q-1)=(j-1) L_{\Omega}-(i-1) L_{M \Omega} \bmod \left(L_{\Omega} L_{M}\right),
$$

which can be also expressed as:

$$
N_{p}(q-1)+F L_{\Omega} L_{M}=\left[(j-1) L_{\Omega}-(i-1) L_{M \Omega}\right],
$$

being $F$ an unknown integer. Equation (44) is a Diophantine equation that allows to obtain the relative positions $(q)$ of all the satellites of the constellation. Once the values of $q$ are computed, it is possible to obtain the along-track distribution of the constellation using Eq. (11).

Moreover, as a second case of study, we deal with constellation that are distributed in several ground-tracks. In this situation, there is no limitation in the selection of the constellation parameters $L_{\Omega}, L_{M}$ and $L_{M \Omega}$ since the constellation is not constrained to a common ground-track, and a direct relation can be performed between Eqs. (3) and (7) to obtain:

$$
\begin{aligned}
t_{k q}-t_{0} & =\frac{T_{c}}{N_{p}}\left[\frac{j-1}{L_{M}}-\frac{L_{M \Omega}(i-1)}{L_{\Omega} L_{M}}\right] \bmod \left(T_{c}\right), \\
\Delta \Omega_{k} & =2 \pi\left[\left(1-\frac{N_{d}}{N_{p}} \frac{L_{M \Omega}}{L_{M}}\right) \frac{i-1}{L_{\Omega}}+\frac{N_{d}}{N_{p}} \frac{j-1}{L_{M}}\right] \bmod (2 \pi),
\end{aligned}
$$

which defines a more general transformation between 2D Lattice Flower Constellations and the formulation provided by Eq. (7).

\subsection{D Necklace Flower Constellations}

2D Necklace Flower Constellations are based on admissible locations defined by the 2D Lattice Flower Constellations formulation. This means that we have to apply the same conditions in $L_{\Omega}, L_{M}$ and $L_{M \Omega}$ in order to obtain a constellation distributed in the same ground-track. On the other hand, the resultant along-track distribution of the constellation can be obtained by introducing Eq. (4) into Eq. (44):

$$
N_{p}(q-1)+E\left(L_{\Omega} L_{M}\right)=\left[\left(\mathcal{G}_{M}-1+S_{M \Omega}\left(\mathcal{G}_{\Omega}-1\right)\right) L_{\Omega}-\left(\mathcal{G}_{\Omega}-1\right) L_{M \Omega}\right]
$$


which is also a Diophantine equation where the value of $q$ for each satellite of the constellation can be obtained. It is important to note that in this case, and since we have introduced necklaces in the formulation, we will only obtain a subset of all the possible values of $q$ that could be generated with the fictitious constellation. In that sense, the values obtained in the transformation are related to the positions where the real satellites of the constellation are located, while the rest of the values of $q$ that are not generated, correspond to empty locations of the configuration.

\subsection{Walker Constellations}

Since Walker Constellations are a subset of 2D Lattice Flower Constellations (Davis et al., 2012), we can benefit of that fact by first relating both formulations. In that sense, the number of satellites of the constellation is $t=L_{\Omega} L_{M}$, the number of orbital planes $p=L_{\Omega}$, and the number of satellites per orbit $t / p=L_{M}$. Moreover, the distribution in right ascension of the ascending node and mean anomaly is obtained as follows:

$$
\begin{aligned}
\Delta \Omega_{i j} & =2 \pi \frac{(i-1)}{p}, \\
\Delta M_{i j} & =2 \pi \frac{p}{t}(j-1)+2 \pi \frac{f}{t}(i-1),
\end{aligned}
$$

or if related with the notation from 2D Lattice Flower Constellations:

$$
\begin{aligned}
\Delta \Omega_{i j} & =\frac{2 \pi}{L_{\Omega}}(i-1), \\
\Delta M_{i j} & =\frac{2 \pi}{L_{M}}(j-1)+\frac{2 \pi}{L_{M}} \frac{f}{L_{\Omega}}(i-1) .
\end{aligned}
$$

By relating Eqs. (48) and (3), it is derived that $L_{M \Omega}=-f \bmod \left(L_{\Omega}\right)$. Moreover, since the limits in definition of the parameter $f \in\{0, \ldots, p-1\}$ and $L_{M \Omega} \in\left\{0, \ldots, L_{\Omega}-1\right\}$, it can be concluded that $L_{M \Omega}=p-1-f$. Therefore, a Walker-Delta Constellation can be defined unequivocally in terms of a $2 \mathrm{D}$ Lattice Flower Constellation. This also means that the conditions to generate a constellation whose satellites are located in the same ground-track is the same as in the case of 2D Lattice Flower Constellations. The same applies to the transformation between Walker-Delta Constellations and the proposed formulation.

\section{Example of application}

In this section we propose an example of nominal design of a repeating groundtrack constellation based on four Earth observation satellites in low Earth 
orbits $\left(N_{s t}=4\right)$ that present the properties of repeating ground-track, sunsynchrony, and frozen condition in the eccentricity vector. These design properties are selected to provide a more stable set of conditions for Earth observation. For this example we assume that all the satellites of the constellation have the same payload, which is based on an optical sensor. This means that satellites will require the same local time at the ascending node to maintain the illumination conditions for all the constellation. In addition, we consider that, due to payload requirements, each satellite must present a repeating groundtrack cycle of 59 orbital revolutions $\left(N_{p}=59\right)$ and four days $\left(N_{d}=4\right)$. Finally, and in order to improve the revisiting time of the constellation, a uniform distribution over the same ground-track is imposed $(k=1)$. Note that a non uniform distribution can be also chosen using the formulation provided by Eq. (24), however, we select a uniform distribution to also be able to relate to the different satellite constellation designs studied in this work.

Table 1 Non-perturbed satellite distribution.

\begin{tabular}{|c|cccc|}
\hline Sat. $(\mathrm{k}, \mathrm{q})$ & 1,1 & 1,2 & 1,3 & 1,4 \\
\hline$\Delta \Omega[\mathrm{deg}]$ & 0.0 & 0.0 & 0.0 & 0.0 \\
$\Delta M[\mathrm{deg}]$ & 0.0 & 270.0 & 180.0 & 90.0 \\
$t_{k q}[$ days] & 0.0 & 1.0 & 2.0 & 3.0 \\
\hline
\end{tabular}

The distribution sought can be directly achieved by a uniform distribution over the ground-track using Eq. (12):

$$
\begin{aligned}
& \Delta \Omega_{q}=-2 \pi N_{d} \frac{(q-1)}{N_{s t}} \bmod (2 \pi)=-2 \pi(q-1) \bmod (2 \pi), \\
& \Delta M_{q}=2 \pi N_{p} \frac{(q-1)}{N_{s t}} \quad \bmod (2 \pi)=\frac{59}{2} \pi(q-1) \bmod (2 \pi),
\end{aligned}
$$

which generates not only a unique ground-track for the constellation, but also a unique inertial orbit since $N_{d}=N_{s t}=4$. Table 1 shows the non-perturbed distribution of the constellation in the right ascension of the ascending node, the mean anomaly and the along-track time distance, where Sat. (k,q) relates to a given spacecraft in the space-track $k$ and position $q$ in that space-track. Note that $t_{k q}$ is also providing the revisiting time of each satellite of the constellation. On the other hand, the same distribution can be obtained by means of the Flower Constellations formulation. In particular, and regarding the original Flower Constellations formulation, an equivalent distribution is obtained imposing $F_{d}=F_{n}=1$, and $F_{h}(g)=0 \quad \forall g \in \mathbb{N}$. Using Eq. (2):

$$
\begin{aligned}
& \Delta \Omega_{g}=\quad-2 \pi \frac{F_{n}}{F_{d}}(g-1) \bmod (2 \pi)=-2 \pi(g-1) \bmod (2 \pi), \\
& \Delta M_{g}=2 \pi \frac{F_{n} N_{p}+F_{d} F_{h}(g)}{F_{d} N_{d}}(g-1) \bmod (2 \pi)=\frac{59}{2} \pi(g-1) \bmod (2 \pi),(50)
\end{aligned}
$$

we can observe that both distributions are completely equivalent if we impose $q=g$. Additionally, and regarding 2D Lattice Flower Constellations, the 
Table 2 Initial positions and velocities of the constellation in the ECEF.

\begin{tabular}{|c|ccc|ccc|}
\hline Sat. $(\mathrm{k}, \mathrm{q})$ & $x[\mathrm{~km}]$ & $y[\mathrm{~km}]$ & $z[\mathrm{~km}]$ & $v_{x}[\mathrm{~km} / \mathrm{s}]$ & $v_{y}[\mathrm{~km} / \mathrm{s}]$ & $v_{z}[\mathrm{~km} / \mathrm{s}]$ \\
\hline 1,1 & 5239.796 & -129.887 & 4668.592 & -4.968 & -1.659 & 5.529 \\
1,2 & 4607.737 & 1190.089 & -5159.020 & 5.721 & -0.472 & 5.001 \\
1,3 & -5251.862 & 127.935 & -4663.414 & 4.959 & 1.658 & -5.529 \\
1,4 & -4618.759 & -1190.801 & 5156.713 & -5.713 & 0.473 & -5.000 \\
\hline
\end{tabular}

equivalent distribution is obtained imposing $L_{\Omega}=1, L_{M}=4$ and $L_{M \Omega}=0$. That way, using Eq. (3):

$$
\begin{aligned}
& \Delta \Omega_{i j}=\quad \frac{2 \pi}{L_{\Omega}}(i-1) \bmod (2 \pi)=0 \bmod (2 \pi), \\
& \Delta M_{i j}=\frac{2 \pi}{L_{M}}(j-1)-\frac{2 \pi}{L_{M}} \frac{L_{M \Omega}}{L_{\Omega}}(i-1) \quad \bmod (2 \pi)=\frac{\pi}{2}(j-1) \quad \bmod (2 \pi),
\end{aligned}
$$

where the relations between $j \in\{1,2,3,4\}$ and $q \in\{1,2,3,4\}$ are provided by Eq. (44):

$$
N_{p}(q-1)+F L_{\Omega} L_{M}=\left[(j-1) L_{\Omega}-(i-1) L_{M \Omega}\right] \quad \Longrightarrow \quad 59(q-1)+4 F=(j-1),
$$

obtaining $j=1 \rightarrow q=1, j=2 \rightarrow q=4, j=3 \rightarrow q=3$ and $j=4 \rightarrow$ $q=2$. The same result is obtained when dealing with 2D Necklace Flower Constellations since all the positions of the constellation are occupied.

However, we are more interested in defining the nominal design of this constellation under the Earth gravitational potential. In particular, we consider a gravitational potential of the Earth (NIMA, 2000) up to 4th order terms (including tesserals). Under these conditions, we first have to define the leading satellite of the constellation. In that sense, a numerical algorithm (in particular the one proposed in Arnas (2018)) is used for the purpose of finding a repeating sun-synchronous frozen orbit under the gravitational model considered in this study. Table 2 shows the initial position and velocity in the ECEF frame of reference of the leading satellite of the constellation (satellite 1,1). Note that this satellite defines the nominal orbit for the whole constellation under the model of gravitational potential of the Earth considered, and also serves as a reference for the satellite distribution.

After the initial state of leading satellite is completely defined, we perform the satellite distribution using Eq. (11) and define the constellation based on the propagation of this leading satellite (see also Table 1 for the alongtrack distribution of the constellation). The initial state of the constellation is presented in Table 2 where the positions and velocities are defined in the ECEF frame of reference. On the other hand, Table 3 shows the distribution of the constellation in osculating elements. One important thing to note is that the inertial orbits of the satellites of the constellation are not exactly the same due to Eq. (16). 
Table 3 Osculating elements of the constellation for epoch (UTC Julian date) 21545.222.

\begin{tabular}{|c|ccc|ccc|}
\hline Sat. $(\mathrm{k}, \mathrm{q})$ & $a[\mathrm{~km}]$ & $e[-]$ & $i[\mathrm{deg}]$ & $\Omega[\mathrm{deg}]$ & $\omega[\mathrm{deg}]$ & $\nu[\mathrm{deg}]$ \\
\hline 1,1 & 7171.935 & 0.021 & 100.056 & 7.700 & 42.498 & 0.000 \\
1,2 & 7166.682 & 0.020 & 99.678 & 3.828 & 311.799 & 359.987 \\
1,3 & 7171.967 & 0.021 & 100.067 & 7.672 & 224.771 & 357.624 \\
1,4 & 7166.697 & 0.020 & 99.686 & 3.824 & 129.712 & 2.153 \\
\hline
\end{tabular}

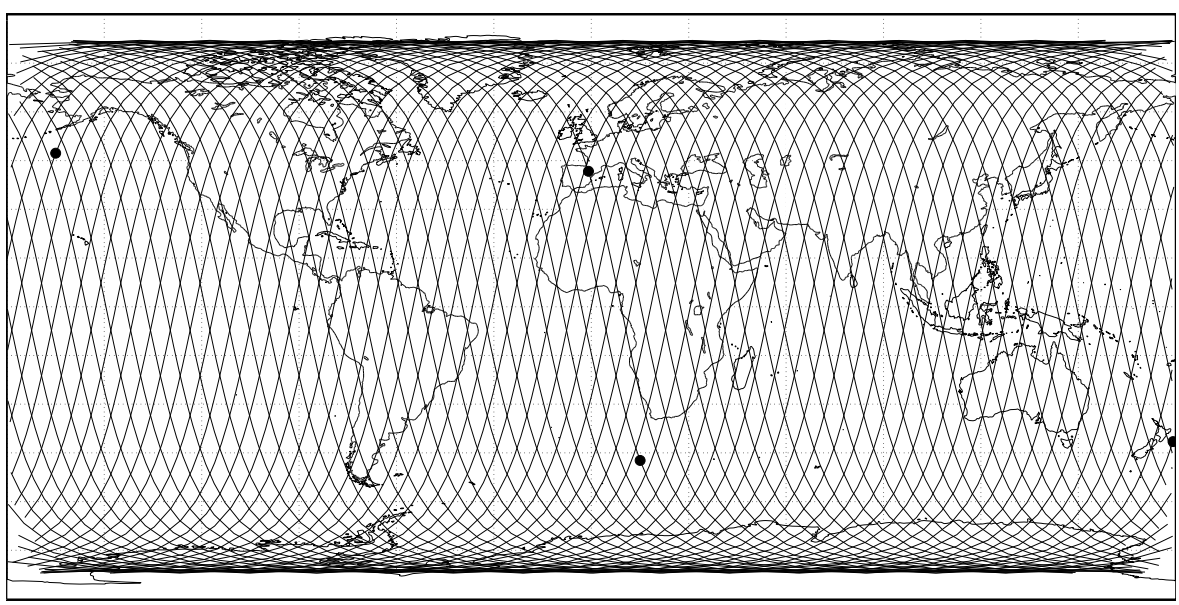

Fig. 1 Ground-track of the constellation.

Table 4 Final positions and velocities of the constellation in the ECEF after one year.

\begin{tabular}{|c|c|c|c|c|c|c|}
\hline & \multicolumn{6}{|c|}{ Computed } \\
\hline & \multicolumn{3}{|c|}{ Position $[\mathrm{km}]$} & \multicolumn{3}{|c|}{ Velocity $[\mathrm{km} / \mathrm{s}]$} \\
\hline Sat. $(k, q)$ & $x$ & $y$ & $z$ & $v_{x}$ & $v_{y}$ & $v_{z}$ \\
\hline 1,1 & 4508.88 & 1189.00 & -5259.75 & 5.82 & -0.44 & 4.88 \\
\hline 1,2 & -5334.07 & 111.27 & -4560.09 & 4.85 & 1.66 & -5.64 \\
\hline 1,3 & -4486.98 & -1186.42 & 5257.85 & -5.83 & 0.44 & -4.88 \\
\hline \multirow[t]{3}{*}{1,4} & 5361.59 & -105.54 & 4538.63 & -4.83 & -1.66 & 5.64 \\
\hline & \multicolumn{6}{|c|}{ Theoretical } \\
\hline & \multicolumn{3}{|c|}{ Position $[\mathrm{km}]$} & \multicolumn{3}{|c|}{ Velocity $[\mathrm{km} / \mathrm{s}]$} \\
\hline Sat. $(k, q)$ & $x$ & $y$ & $z$ & $v_{x}$ & $v_{y}$ & $v_{z}$ \\
\hline 1,1 & 4508.88 & 1189.00 & -5259.75 & 5.82 & -0.44 & 4.88 \\
\hline 1,2 & -5334.00 & 111.26 & -4560.18 & 4.85 & 1.66 & -5.64 \\
\hline 1,3 & -4487.16 & -1186.47 & 5257.68 & -5.83 & 0.44 & -4.88 \\
\hline 1,4 & 5361.35 & -105.51 & 4538.92 & -4.83 & -1.66 & 5.64 \\
\hline
\end{tabular}

Figure 1 shows the ground-track of the constellation for a propagation of 4 days. As it can be seen, all four satellites share the same ground-track, which is closed, achieving the ground-track property for the whole constellation. This state has been achieved even with the perturbation produced by the Earth gravitational potential, obtaining a repeating ground-track property that can be maintained for months (and for the perturbation considered) without orbital maneuvers. In particular, a propagation of one year was performed using this configuration and an adaptable time step. Table 4 shows the position 
and velocity of the constellation after this propagation (column "computed"). Moreover, in order to show the evolution of the relative distribution itself, an additional computation (column "theoretical") is done. This computation was performed by taking the values of position and velocity from the first satellite $(1,1)$ after the one year propagation as the reference for the constellation, and performing the constellation distribution from them, that is, the positions and velocities of the this "theoretical" constellation are computed using the distribution defined by Eq. (11). As it can be seen, the difference between both results is minimal, being these differences a consequence of the error accumulation after one year of propagation of the constellation. It is important to emphasize that if other orbital perturbations are considered, the space-track of the constellation will change, and thus, orbital maneuvers will be required to be applied to correct that situation.

\section{Conclusion}

This work presents a methodology to perform the nominal design of repeating ground-track constellations under the effect of the perturbation produced by the Earth gravitational potential. In particular, we provide a new mathematical formulation to define these systems, and analyze the long term dynamic of the resultant constellations. The general idea of this procedure is to define the constellation distribution directly in the ECEF frame of reference using the along-track and cross-track time distances between satellites. That way, it is possible to include the effects of these perturbations directly in the nominal definition of the constellation, being able to maintain the along-track distribution of the constellation during the dynamic of the system. This methodology is based on the definition of a set of leading satellites, one per each different space-track of the constellation, that are used in order to generate the set of perturbed space-tracks in which the satellite distribution is defined. Following this procedure, these reference space-tracks allow to distribute satellites in such a way that the constellation along-track distribution is maintained under the perturbation produced by the Earth gravitational potential. In that sense, we show that some additional considerations have to be taken into account. In particular, the satellite distribution must consider the combined effect of the mean anomaly and the variation of the argument of perigee in order to define a time invariant distribution under these orbital perturbations. Moreover, the secular variation of the right ascension of the ascending node and the mean anomaly of the leading satellite have to be included in the nominal distribution of the constellation.

Additionally, a transformation between Flower Constellations (including Lattice and Necklace formulations), Walker Constellations, and and a relative to Earth formulation is introduced. This allows, for instance, to obtain the relative distribution in along-track and cross-track distances of Flower Constellations in the ECEF frame of reference. The most important application of these transformations is to be able to extend the interesting properties in the 
ECEF frame of reference of the formulation presented in this work to other satellite constellation designs. This means that, with these transformations, and following the design procedure presented, it is possible to define the nominal design of any Flower Constellation under the effect of the perturbation produced by the Earth gravitational potential. In that sense, Flower Constellations were selected since they represent a generalization of the most common satellite constellation designs currently in use. Moreover, this set of transformations can be used to compute the revisiting times between the subsatellite points of repeating ground-track constellations by the sole use of integer operations, since the along-track distribution is provided directly by the proposed mathematical formulation.

Finally, an example of application for a LEO Earth observation constellation is presented, where we show how the distribution can be maintained using this methodology for long periods of time (more than a year) under a $4 \times 4$ model of the Earth gravitational potential. In this example we deal with sun-synchronous orbits that have the frozen eccentricity condition, since this is a wide-spread design for Earth observation missions, and show their relations with all the formulations used in this work.

\section{Funding Sources}

The work of David Arnas and Daniel Casanova was supported by the Spanish Ministry of Economy and Competitiveness, Project no. ESP2017-87113-R (AEI/FEDER, UE); and by the Aragon Government and European Social Fund (group E24_17R).

\section{Conflict of interest}

The authors declare that they have no conflict of interest.

\section{References}

Arnas, D.: Necklace Flower Constellations. Thesis dissertation, Universidad de Zaragoza, 2018.

Arnas, D., Casanova, D., Tresaco, E.: Relative and absolute station-keeping for two-dimensional-lattice flower constellations. J. Guid. Control Dyn. 39(11), 2596-2602 (2016a). doi:10.2514/1.G000358.

Arnas, D., Casanova, D., Tresaco, E.: Corrections on repeating ground-track orbits and their applications in satellite constellation design. Adv. Astronaut. Sci., 158, 2823-2840 (2016b). ISBN: 978-0-87703-634-0.

Arnas, D., Casanova, D., Tresaco, E.: Time distributions in satellite constellation design. Celest. Mech. Dyn. Astron. 128(2), 197-219 (2017a). doi: 10.1007/s10569-016-9747-3. 
Arnas, D., Casanova, D., Tresaco, E.: 2D Necklace Flower Constellations. Acta Astronaut. 142, 18-28 (2018). doi: 10.1016/j.actaastro.2017.10.017.

Arnas, D., Casanova, D., Tresaco, E., Mortari, D.: 3-Dimensional Necklace Flower Constellations. Celest. Mech. Dyn. Astron. 129(4), 433-448 (2017b). doi: $10.1007 /$ s10569-017-9789-1.

Avendaño, M.E., Davis, J.J., Mortari, D.: The 2-D lattice theory of flower constellations. Celest. Mech. Dyn. Astron. 116(4), 325-337 (2013). doi:10.1007/s10569-013-9493-8.

Avendaño, M. E., Mortari, D.: New Insights on Flower Constellations Theory. J. IEEE Trans. Aerosp. Electron. Syst. 48(2), 1018-1030 (2012). doi: 10.1109/TAES.2012.6178046.

Ballard, A.H.: Rossete constellations of Earth satellites. J. IEEE Trans. Aerosp. Electron. Syst., 5, 656-673, (1980). doi: 10.1109/TAES.1980.308932.

Beste, D.C.: Design of satellites constellations for optimal continuous coverage. J. IEEE Trans. Aerosp. Electron. Syst., 3, 466-473, (1978). doi: 10.1109/TAES.1978.308608.

Casanova, D., Avendaño, M.E., Mortari, D.: Design of Flower Constellations using Necklaces. J. IEEE Trans. Aerosp. Electron. Syst. 50(2), 1347-1358 (2014a). doi: 10.1109/TAES.2014.120269.

Casanova, D., Avendaño, M.E., Mortari, D.: Seeking GDOP-optimal Flower Constellations for global coverage problems through evolutionary algorithms. Journal of Aerospace Science and Technology 39, 331-337 (2014b). doi: 10.1016/j.ast.2014.09.017.

Casanova, D., Avendaño, M.E., Tresaco, E.: Lattice-preserving flower constellations under J2 perturbations. Celest. Mech. Dyn. Astron. 121(1), 83-100 (2014c). doi:10.1007/s10569-014-9583-2.

Davis, J.J., Mortari, D.: Reducing Walker, Flower, and Streets-of-Coverage Constellations to a Single Constellation Design Framework. Adv. Astronaut. Sci., 143, 697-712 (2012). ISBN: 978-0-87703-581-7.

Davis, J.J., Avendaño, M.E., Mortari, D.: The 3-D lattice theory of flower constellations. Celest. Mech. Dyn. Astron. 116(4), 339-356 (2013). doi:10.1007/s10569-013-9494-7.

Draim, J. E.: A common-period four-satellite continuous global coverage constellation. J. Guid. Control Dyn. 10(5), 492-499 (1987). ISSN 0731-5090.

Dufour, F.: Coverage optimization of elliptical satellite constellations with an extended satellite triplet method, 54th International Astronautical Congress of the International Astronautical Federation, the International Academy of Astronautics, and the International Institute of Space Law, International Astronautical Congress (IAF), A-3. doi: 10.2514/6.IAC-03-A.3.02 (2003).

Lo, M. W.: Satellite-Constellation Design. Comput. Sci. Eng., 1(1), 58-67, (1999). doi: 10.2514/1.35369.

Luders, R.: Satellite networks for continuous zonal coverage. ARS J., 31(2), 179-184, (1961). doi: 10.2514/8.5422.

Mordell, L. J.: Diophantine equations. Academic Press, (1969). ISBN: 9780080873428 
Mortari, D., Avendaño, M. E., Lee S.: J2-Propelled Orbits and Constellations. J. Guid. Control Dyn. 37(5), 1701-1706 (2014). doi: 10.2514/1.G000363.

Mortari, D., Wilkins, M.P., Bruccoleri, C.: The flower constellations. J. Astronaut. Sci. Am. Astronaut. Soc. 52(1-2), 107-127 (2004).

Mozhaev, G.V.: The problem of continuous Earth coverage and the Kinematically regular satellite networks. Cosmic Res+, 11, 755, (1973).

National Imagery and Mapping Agency: World Geodetic System 1984, Third Edition. National Imagery and Mapping Agency (2000).

Ulybyshev, Y.: Satellite constellation design for complex coverage. J. of Spacecraft Rockets, 45(4), 843-849, (2008). doi: 10.2514/1.35369.

Wagner, C.: A Prograde Geosat Exact Repeat Mission? J. Astronaut. Sci. 39, 313-326 (1991).

Walker, J.G.: Satellite constellations. J. Br. Interplanet. Soc. 37, 559-572 (1984). ISSN 0007-084X.

Wook, S., Kronig, L.G., Ivanov, A.B., Weck, O.L.: Satellite constellation design algorithm for remote sensing of diurnal cycles phenomena. Adv. Space Res. 62, 2529-2550 (2018). doi: 10.1016/j.asr.2018.07.012. 